\title{
Correction: Artificial Intelligence-Based Prediction of Lung Cancer Risk Using Nonimaging Electronic Medical Records: Deep Learning Approach
}

Marvin Chia-Han Yeh ${ }^{1,2}, \mathrm{MD}, \mathrm{PhD}$; Yu-Hsiang Wang ${ }^{3}$, MD; Hsuan-Chia Yang ${ }^{4,5}, \mathrm{PhD}$; Kuan-Jen Bai ${ }^{6,7}$, MD; Hsiao-Han Wang ${ }^{1,2,4,}, \mathrm{MD}$; Yu-Chuan Jack $\mathrm{Li}^{1,2,4,5,9 *}, \mathrm{MD}, \mathrm{PhD}$

${ }^{1}$ Department of Dermatology, Wan Fang Hospital, Taipei Medical University, Taipei, Taiwan

${ }^{2}$ Research Center of Big Data and Meta-analysis, Wan Fang Hospital, Taipei Medical University, Taipei, Taiwan

${ }^{3}$ School of Medicine, Taipei Medical University, Taipei, Taiwan

${ }^{4}$ Graduate Institute of Biomedical Informatics, College of Medical Science and Technology, Taipei Medical University, Taipei, Taiwan

${ }^{5}$ International Center for Health Information Technology, Taipei Medical University, Taipei, Taiwan

${ }^{6}$ Division of Pulmonary Medicine, Department of Internal Medicine, Wan Fang Hospital, Taipei Medical University, Taipei, Taiwan

${ }^{7}$ School of Respiratory Therapy, College of Medicine, Taipei Medical University, Taipei, Taiwan

${ }^{8}$ Pulmonary Research Center, Wan Fang Hospital, Taipei Medical University, Taipei, Taiwan

${ }^{9}$ Department of Dermatology, School of Medicine, Taipei Medical University, Taipei, Taiwan

*these authors contributed equally

\section{Corresponding Author:}

Yu-Chuan Jack Li, MD, PhD

Department of Dermatology

Wan Fang Hospital

Taipei Medical University

No 111, Section 3, Xinglong Road

Wenshan District

Taipei, 116

Taiwan

Phone: 88629307930 ext 2980

Email: jaak88@gmail.com

\section{Related Article:}

Correction of: https://www.jmir.org/2021/8/e26256

(J Med Internet Res 2021;23(10):e33519) doi: 10.2196/33519

Correction: Artificial Intelligence-Based Prediction of Lung Cancer Risk Using Nonimaging Electronic Medical Records: Deep Learning Approach

In "Artificial Intelligence-Based Prediction of Lung Cancer Risk Using Nonimaging Electronic Medical Records: Deep Learning Approach" (J Med Internet Res 2021;23(8):e26256), two errors were noted.

Due to a system error, the name of one author, Marvin Chia-Han Yeh, was replaced with the name of another author on the paper, Hsuan-Chia Yang. As well, the formatting of the author name "Yu-Chuan (Jack) Li" has been changed to "Yu-Chuan Jack Li" in the corrected version of the paper.

In the originally published paper, the order of authors was listed as follows:

Hsuan-Chia Yang, Yu-Hsiang Wang, Hsuan-Chia Yang, Kuan-Jen Bai, Hsiao-Han Wang, Yu-Chuan (Jack) Li.
This has been corrected to:

Marvin Chia-Han Yeh, Yu-Hsiang Wang, Hsuan-Chia Yang, Kuan-Jen Bai, Hsiao-Han Wang, Yu-Chuan Jack Li.

In the originally published paper, the ORCID of author Hsuan-Chia Yang was incorrectly published as follows:

$$
\text { 0000-0001-6710-4435 }
$$

This has been corrected to:

$$
\text { 0000-0001-9198-0697 }
$$

The correction will appear in the online version of the paper on the JMIR Publications website on October 15, 2021, together with the publication of this correction notice. Because this was made after submission to PubMed, PubMed Central, and other full-text repositories, the corrected article has also been resubmitted to those repositories. 


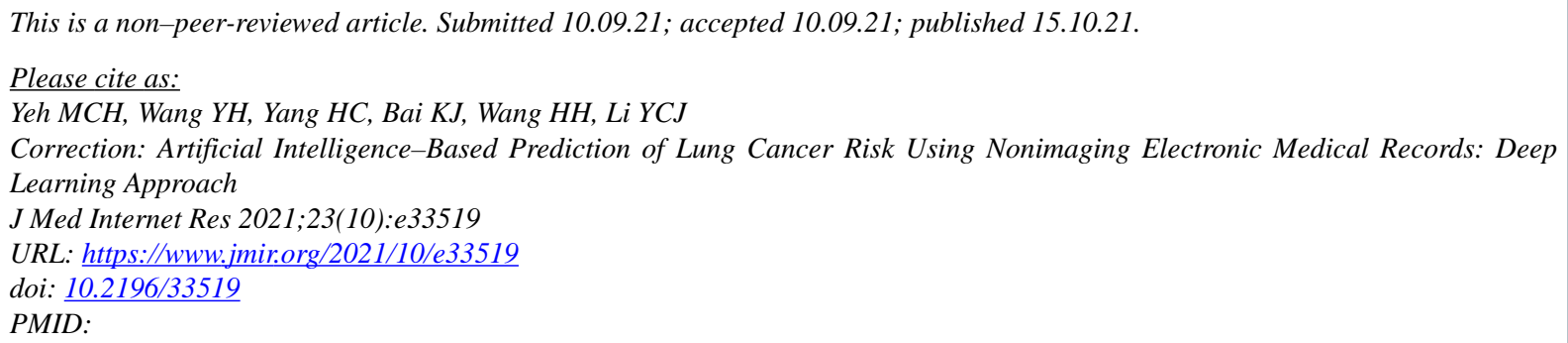

CMarvin Chia-Han Yeh, Yu-Hsiang Wang, Hsuan-Chia Yang, Kuan-Jen Bai, Hsiao-Han Wang, Yu-Chuan Jack Li. Originally published in the Journal of Medical Internet Research (https://www.jmir.org), 15.10.2021. This is an open-access article distributed under the terms of the Creative Commons Attribution License (https://creativecommons.org/licenses/by/4.0/), which permits unrestricted use, distribution, and reproduction in any medium, provided the original work, first published in the Journal of Medical Internet Research, is properly cited. The complete bibliographic information, a link to the original publication on https://www.jmir.org/, as well as this copyright and license information must be included. 